\title{
EFEITO DO TREINAMENTO SOBRE A EFICIÊNCIA VENTILATÓRIA DE INDIVÍDUOS SAUDÁVEIS
}

\author{
EFFECT OF TRAINING ON VENTILATORY EFFICIENCY IN HEALTHY SUBJECTS
}

EFECTO DEL ENTRENAMIENTO SOBRE LA EFICIENCIA VENTILATORIA

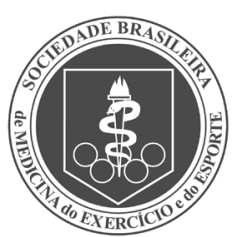

Artigo Original

DE INDIVIDUOS SALUDABLES

Deborah Sauer ${ }^{1}$
(Professora de Educação Física)
Anselmo José Perez ${ }^{1}$
(Professor de Educação Física)
Luciana Carletti $^{1}$
(Professora de Educação Física)

1. Laboratório de Fisiologia do Exercício (LAFEX) da Universidade Federal do Espírito Santo (UFES), Vitória, ES, Brasil.

\section{Correspondência:}

Rua Doutor Cyro Lopes Pereira, 215/101, Jardim da Penha, 29060-020 Vitória, ES, Brasil.

sauerdeborah@hotmail.com.

\section{RESUMO}

Introdução: Diversos índices de eficiência ventilatória (EV) têm fornecido uma medida extra para avaliação do condicionamento cardiorrespiratório em adição ao consumo de oxigênio $\left(\mathrm{VO}_{2}\right)$ no pico do exercício e no nível do limiar ventilatório $\left(\mathrm{VO}_{2} \mathrm{LV}\right)$. Em indivíduos com insuficiência cardíaca já foi demonstrado que há aumento da EV após treinamento. No entanto, a sensibilidade dessa medida para avaliar o efeito do treinamento em indivíduos saudáveis foi pouco estudada. Objetivo: Testar a hipótese de que um programa de treinamento delineado para melhorar a condição aeróbia, também exerça alterações na eficiência ventilatória em indivíduos saudáveis. Métodos: 48 homens, aparentemente saudáveis e ativos (24 \pm 5 anos), foram submetidos a um teste cardiopulmonar de exercício (TCPE), antes e após 13 semanas de treinamento aeróbio, realizado três vezes por semana, durante 30 minutos com a intensidade inicial de 60-65\% da $F C_{\text {máx }}$ gradualmente aumentada até o fim do programa para 85-90\% da $\mathrm{FC}_{\max }$. Os parâmetros avaliados incluíram: $\mathrm{VO}_{2 \text { pico, }} \mathrm{VO}_{2}$ no $\mathrm{LV}$ e EV determinada através do cálculo do slope da relação entre a ventilação e a produção de dióxido de carbono, por meio de regressão linear. Resultados: Houve um aumento de $12,5 \%$ no VO $2 \mathrm{LV}\left(30,4 \pm 4,5\right.$ vs. $\left.34,2 \pm 4,9 \mathrm{ml}^{\mathrm{kg}}{ }^{-1} \cdot \mathrm{min}^{-1}, \mathrm{p}<0,05\right) \mathrm{e}$ de $10,9 \%$ no $\mathrm{VO}_{2 \text { pico }}\left(53,2 \pm 8,3 \mathrm{vs.} 59 \pm 9,9 \mathrm{ml} . \mathrm{kg}^{-1} \cdot \mathrm{min}^{-1}, \mathrm{p}<0,05\right)$, acompanhado de uma redução de $4,1 \%$ no slope

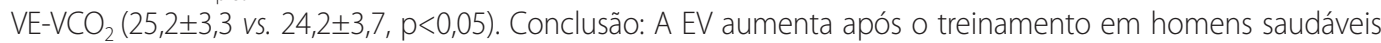
sugerindo que o slope da relação $\mathrm{VE}-\mathrm{VCO}_{2}$ pode ser utilizado de forma adicional na monitoração dos efeitos do treinamento, complementando a interpretação da integração cardiorrespiratória do TCPE.

Palavras-chave: aptidão física, ventilação pulmonar, consumo de oxigênio, teste de esforço, exercício.

\section{ABSTRACT}

Introduction: Several indices of ventilatory efficiency (VE) have provided an extra measure to assess cardiorespiratory fitness in addition to oxygen uptake $\left(\mathrm{VO}_{2}\right)$ at peak exercise and at the level of ventilatory threshold $\left(\mathrm{VO}_{2} \mathrm{VT}\right)$. In patients with heart failure has been demonstrated that there is increase of ventilatory efficiency after training. However, the sensitivity of this measure to assess the effect of training in health subjects has been little studied. Objective: To test the hypothesis that a training program designed to improve aerobic fitness also changes the ventilatory efficiency in healthy subjects. Methods: 48 men seemingly healthy and active (24 \pm 5 years) underwent a cardiopulmonary exercise test (CPET) before and after 13 weeks of aerobic training, performed three times a week for 30 minutes with the initial intensity 60$65 \%$ of maximum heart rate $\left(H R_{\max }\right)$ gradually increased until the end of program for $85-90 \%$ of $H R_{\max }$. The parameters evaluated included: $\mathrm{VO}_{2 \text { peak }} \mathrm{VO}_{2} \mathrm{VT}$ and ventilatory efficiency that was determined by calculating the slope of the relationship between ventilation and production of carbon dioxide by linear regression. Results: There was a 12.5\% increase in

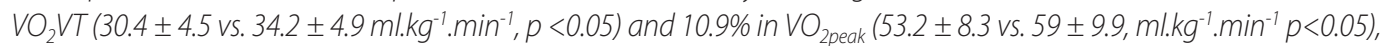
accompanied by a $4.1 \%$ reduction in VE-VCO ${ }_{2}$ slope $(25.2 \pm 3.3$ vs. $24.2 \pm 3.7, p<0.05)$. Conclusion: Ventilatory efficiency increases after training in healthy men suggesting that the slope of the relationship VE-V $\mathrm{CO}_{2}$ can be used in addition to the monitoring of the effects of training, complementing the interpretation of cardiorespiratory integration of CEPT.

Keywords: Physical fitness, pulmonary ventilation, oxygen consumption, exercise test, exercise.

\section{RESUMEN}

Introducción: Diversos índices de eficiencia ventilatoria (EV) han suministrado una medida extra para evaluación del condicionamiento cardiorrespiratorio en adición al consumo de oxígeno $\left(\mathrm{VO}_{2}\right)$ en el pico del ejercicio y en el nivel del umbral ventilatorio $\left(\mathrm{VO}_{2} \mathrm{LV}\right.$ ). En individuos con insuficiencia cardíaca ya fue demostrado que hay aumento de la EV después del entrenamiento. Sin embargo, la sensibilidad de esa medida para evaluar el efecto del entrenamiento en individuos saludables fue poco estudiada. Objetivo: Probar la hipótesis de que un programa de entrenamiento delineado para mejorar la condición aeróbica, que también ejerza alteraciones en la eficiencia ventilatoria en individuos saludables. Métodos: 48 hombres, aparentemente saludables y activos (24 \pm 5 años), fueron sometidos a un test cardiopulmonar de ejercicio (TCPE), antes y después de 13 semanas de entrenamiento aeróbico, realizado tres veces por semana, durante 30 minutos con la intensidad inicial de 60-65\% de la $F C_{\max }$ gradualmente aumentada hasta el fin del programa para 85-90\% de la $F C_{\max }$ Los parámetros evaluados incluyeron: $\mathrm{VO}_{2 p i c a} \mathrm{VO}_{2}$ en el LV y EV determinada a través del cálculo del slope de la relación entre la ventilación y la producción de dióxido de carbono, 
por medio de regresión lineal. Resultados: Hubo un aumento de 12,5\% en el VO $\mathrm{LV}_{2}\left(30,4 \pm 4,5 \mathrm{vs} .34,2 \pm 4,9 \mathrm{ml} \cdot \mathrm{kg}^{-1} \cdot \mathrm{min}^{-1}\right.$, $p<0,05)$ y de $10,9 \%$ en el $\mathrm{VO}_{2 \text { pico }}\left(53,2 \pm 8,3 \mathrm{vs} .59 \pm 9,9 \mathrm{ml} \cdot \mathrm{kg}^{-1} \cdot \mathrm{min}^{-1}, p<0,05\right)$, acompañado de una reducción de $4,1 \%$ en

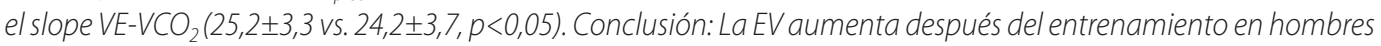
saludables sugiriendo que el slope de la relación VE-VCO 2 puede ser utilizado de forma adicional en el monitoreo de los efectos del entrenamiento, complementando la interpretación de la integración cardiorrespiratoria del TCPE.

Palabras clave: aptitud física, ventilación pulmonar, consumo de oxígeno, test de esfuerzo, ejercicio.

\section{INTRODUÇÃO}

$\mathrm{O}$ consumo de oxigênio $\left(\mathrm{VO}_{2}\right)$ no pico do exercício e no limiar ventilatório (LV) são os indicadores de referência para mensurar a aptidão cardiorrespiratória. Em adição, diversos índices de eficiência ventilatória (EV) obtidos por meio do teste cardiopulmonar de exercício (TCPE), têm fornecido uma medida extra utilizada na avaliação de indivíduos saudáveis ${ }^{1-6}$ e principalmente de pacientes com insuficiência cardíaca (IC) ${ }^{7}$.

A determinação da EV pode ser realizada através do cálculo do slope da relação entre a ventilação (VE) e a produção de dióxido de carbono $\left(\mathrm{VCO}_{2}\right)$ utilizando pontos de dados do início do TCPE até o LV ${ }^{1,4,8}$, ponto de compensação respiratória $(P C R)^{2,3,9,10}$ e $V_{2}$ max/pico $^{5,6,9-16}$, bem como por meio da razão VE $\mathrm{VCO}_{2}$ em pontos do TCPE que habitualmente incluem o LV3,4,17, $\mathrm{PCR}$ e/ou $\mathrm{VO}_{2 \text { max/pico }}$ 4,16,18,19.

Os valores elevados do slope VE-V $\mathrm{CO}_{2}$ indicam que o pulmão precisa ser ventilado mais vezes para eliminar uma dada quantidade de $\mathrm{CO}_{2}$. Esse impulso ventilatório aumentado está presente no perfil de uma série de doenças cardíacas ${ }^{18}$ e pulmonares e por isso, a mensuração da EV tem sido útil na avaliação e prognóstico desses pacientes ${ }^{9,11,12}$.

O valor normal do slope VE-VCO 2 é abaixo de $30^{3,7}$, sendo que valores acima disso caracterizam uma resposta anormal frequentemente associada à gravidade da $\mathrm{IC}^{7,12}$. Em atletas, foi encontrada uma correlação fraca entre o slope VE- $\mathrm{VCO}_{2}$ e VO $\mathrm{Vmax}^{5,6}$, sugerindo uma associação limitada entre EV e desempenho. Por outro lado, na IC a elevação do slope VE-VCO $\mathrm{V}_{2}$ está inversamente relacionada com a capacidade aeróbia avaliada pelo $\mathrm{VO}_{2 \max }{ }^{11,12}$. Além disso, já foi demonstrado nesses indivíduos aumento da EV após treinamento $8,10,13-16,19,20$, o que tem sido atribuído à melhora do metabolismo oxidativo dos músculos esqueléticos, aumento do débito cardíaco e atraso na acidose metabólica ${ }^{10,13,19}$. Entretanto, os poucos estudos que avaliaram os efeitos do treinamento sobre o slope $\mathrm{VE}-\mathrm{VCO}_{2}$ de indivíduos saudáveis, não encontraram alterações ${ }^{1,4}$. Assim, apesar da relevância clínica da EV para pacientes com IC, a sensibilidade dessa medida para a avaliação de indivíduos saudáveis ainda foi pouco estudada. Considerando que parte das adaptações ao treinamento associadas ao aumento da EV em pacientes com IC, também ocorre em pessoas saudáveis, esse trabalho teve como finalidade testar a hipótese de que um programa de treinamento delineado para melhorar a condição aeróbia, também exerça alterações na EV.

\section{MÉTODOS}

A amostra foi composta por 48 homens aparentemente saudáveis, com média de idade de $24 \pm 5$ anos. $O$ estudo foi aprovado pelo Comitê de Ética do Centro de Ciências da Saúde - CCS/UFES (Parecer n 1102/98). Todos os objetivos e procedimentos da pesquisa foram explicados detalhadamente aos indivíduos, que concordaram com a participação no estudo, assinando um termo de consentimento livre e esclarecido. Os indivíduos foram considerados como ativos, de acordo com anamnese realizada previamente ao estudo, porém nenhum deles tinha participação sistemática em programas de exercícios. Como critérios de exclusão foram considerados: a evidência de comprometimentos osteoarticulares, doenças cardiovasculares ou metabólicas, participação em outro programa de exercícios durante o treinamento, terapia farmacológica com betabloqueadores e uma frequência inferior a 85\% do total das sessões de treinamento.
A massa corporal e a estatura foram medidas em uma balança com toesa, da marca Welmy (Santa Bárbara d’Oeste, Brasil) com precisão de 0,1 kg e 0,1 cm, respectivamente. A partir desses dados foi possível calcular o índice de massa corporal (IMC, kg/m²).

\section{Teste Cardiopulmonar de Exercício (TCPE)}

Em primeiro lugar os sujeitos eram submetidos a um eletrocardiograma (ECG) de repouso (sistema TEB, modelo SM 400, Porto Alegre, RS, Brasil), nas 12 derivações convencionais. Em seguida, era acoplado um pneumotacômetro na cavidade oral dos participantes, acompanhado de um clip nasal para as medidas ventilatórias. O avaliado ficava em pé durante três minutos para execução das medidas referentes ao pré-esforço, e após essa etapa era realizado o TCPE por meio do protocolo de Bruce ${ }^{21}$ com monitoração contínua da frequência cardíaca (FC) através do intervalo R-R obtido pelo ECG, utilizando derivações simultâneas em MC5, D2M e V2M. Foram realizados dois testes, um imediatamente antes do início do programa de treinamento (teste 1 - T1), e outro após o término (teste 2 - T2). Ambos os testes foram executados em ambiente com temperatura controlada e no mesmo período do dia.

Os critérios para aceitar o teste como máximo incluíram: a) exaustão voluntária; b) $\mathrm{FC}_{\max }$ atingida no teste estar pelo menos a 90\% da prevista para idade (220-idade); c) razão de troca respiratória (RTR) igual ou acima de 1,122.

A mensuração dos volumes de oxigênio e de gás carbônico (circuito aberto) foi feita pelo analisador de gases AeroSport TEEM 100 Total Metabolic Analysis System (Aerosport, Ann Arbor, MI, USA) ${ }^{23}$, com pneumotacômetro de fluxo médio. A amostragem ventilatória dos gases expirados foi calculada em média de 20 segundos, sendo a análise realizada com ajuda do programa Aerograph, V 2.06. A unidade do Aerosport TEEM 100 foi calibrada pelo método GASCAL+ZERO (calibração de circuito-fechado), através de gás de calibração (cilindro de $\% \mathrm{O}_{2}$ e $\% \mathrm{CO}_{2}$ original, fornecido pelo fabricante).

O limiar ventilatório (LV) foi determinado sempre pelos mesmos dois avaliadores e de forma independente, utilizando como critério o método visual da perda da linearidade da relação entre o consumo de oxigênio e a produção de dióxido de carbono (V-Slope ${ }^{24}$. Para apoiar a confirmação do LV pelo V-Slope, também foi utilizado o ponto mais baixo do equivalente ventilatório de oxigênio $\left(\mathrm{VE} / \mathrm{VO}_{2}\right)$ antes da elevação sustentada e sem aumento concomitante do equivalente ventilatório de dióxido de carbono $\left(\mathrm{VE} / \mathrm{NCO}_{2}\right)^{25}$.

A eficiência ventilatória (EV) foi determinada para cada indivíduo através do cálculo do slope da relação entre a VE (BTPS) e a VCO (STPD), por meio de análise de regressão linear (Microsoft Office Excel), utilizando os pontos de dados do início do TCPE até o $\mathrm{VO}_{2 \max }$ sendo a média do intervalo de amostragem ventilatória de 20 segundos.

\section{Protocolo de treinamento}

O programa de treinamento foi realizado por meio de caminhada e/ou corrida em pista, durante 13 semanas, três vezes por semana, em sessões de 50 minutos, sendo 10 minutos de aquecimento, 30 minutos de estímulo na zona alvo e 10 minutos de alongamento. A intensidade inicial correspondeu a 60-65\% da $F C_{\max }$ medida e foi gradualmente aumentada $5 \%$ ao longo das semanas de treinamento, chegando a 
85-90\% da $\mathrm{FC}_{\max }$ medida, na última semana. O controle da intensidade foi efetuado por meio de monitores de FC da marca Polar, modelos Beat, Accurex e Accurex plus, (Kempele, Finlândia).

\section{Análise estatística}

Após o teste de normalidade de Kolmogorov-Smirnov, as variáveis $\mathrm{VO}_{\text {2pico, }} \mathrm{VO}_{2}$ no LV e EV foram submetidas a teste $t$ de Student para dados pareados visando determinar as diferenças antes e após o treinamento. Foi adotado um nível de significância de 5\%. Os dados são apresentados em média \pm desvio padrão (DP), sendo que os cálculos estatísticos foram efetuados com o programa Sigma Stat, versão 3.5, Systat Software Inc. (San José, Estados Unidos).

\section{RESULTADOS}

As características antropométricas dos sujeitos são apresentadas na tabela 1, onde os valores do índice de massa corporal (IMC) denotam a ausência de sobrepeso ou obesidade.

Na tabela 2 são exibidos os dados referentes às variáveis avaliadas por meio do TCPE. Após o treinamento houve um aumento significativo

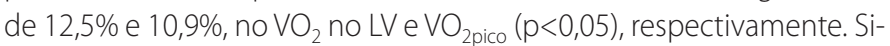
milarmente houve uma redução significativa de 4,1\% no slope $\mathrm{VE}-\mathrm{VCO}_{2}$, $(p<0,05)$ o que indica um aumento da EV como efeito do treinamento.

Tabela 1. Caracterização antropométrica e etária.

\begin{tabular}{c|c}
\hline Parâmetros & Média $(\mathbf{n = 4 8 )}$ \\
\hline Idade (anos) & $24 \pm 5$ \\
\hline Massa Corporal $(\mathrm{kg})$ & $70 \pm 12$ \\
\hline Estatura $(\mathrm{m})$ & $1,77 \pm 0,06$ \\
\hline IMC $\left(\mathrm{kg} / \mathrm{m}^{2}\right)$ & $22 \pm 3$ \\
\hline
\end{tabular}

Valores expressos como média \pm desvio padrão; IMC: índice de massa corporal.

Tabela 2. Efeito do treinamento no $\mathrm{VO}_{2 \text { pico, }}$ limiar ventilatório e eficiência ventilatória.

\begin{tabular}{c|c|c}
\hline Variável & T1 $(\mathbf{n}=\mathbf{4 8})$ & T2 $(\mathbf{n = 4 8 )}$ \\
\hline $\mathrm{VO}_{2 \text { pico }}\left(\mathrm{ml}^{\mathrm{kg}} \mathrm{kg}^{-1} \cdot \mathrm{min}^{-1}\right)$ & $53,2 \pm 8,3$ & $59 \pm 9,9^{*}$ \\
\hline $\mathrm{VO}_{2} \mathrm{LV}\left(\mathrm{ml}_{\mathrm{kg}} \mathrm{kg}^{-1} \cdot \mathrm{min}^{-1}\right)$ & $30,4 \pm 4,5$ & $34,2 \pm 4,9^{*}$ \\
\hline $\mathrm{VE}-\mathrm{VCO}_{2}$ slope & $25,2 \pm 3,3$ & $24,2 \pm 3,7^{*}$ \\
\hline
\end{tabular}

Valores expressos como média \pm desvio padrăo; $p<0,05$; *diferença significativa em relaçăo ao pré-treinamento (Teste $t$ de student para dados pareados); $T 1$ : teste $1 ; \mathrm{T} 2$ : teste $2 ; \mathrm{VO}_{2 \text { pico: }}$ consumo de oxigênio de pico; $\mathrm{VO}_{2} \mathrm{LV}$ : consumo de oxigênio no limiar ventilatório; $\mathrm{VE}-\mathrm{VCO}_{2}$ slope: cálculo do slope da relação entre a ventilação e a produção de dióxido de carbono.

\section{DISCUSSÃO}

A hipótese de que um programa de treinamento delineado para melhorar a condição aeróbia refletida pelo $\mathrm{VO}_{\text {2pico }}$ e LV seria acompanhado de alterações na EV foi confirmada no presente estudo. Foi encontrado um aumento no $\mathrm{VO}_{2 \text { pico }}$ e $\mathrm{VO}_{2}$ no $\mathrm{LV}$, em paralelo com um decréscimo no slope $\mathrm{VE}-\mathrm{VCO}_{2}$, sugerindo que após o treinamento houve uma redução do impulso ventilatório requerido para eliminar uma dada quantidade de $\mathrm{CO}_{2}$ durante o TCPE. Esses achados estão em contraste com outros estudos que avaliaram os efeitos do treinamento na EV de indivíduos saudáveis ${ }^{1,4}$.

Clark et al. ${ }^{1}$ por exemplo, não encontraram alterações na EV de 27 indivíduos saudáveis e sedentários, após 19 semanas de treinamento aeróbio, realizado três vezes por semana, durante 40 minutos, apesar de um aumento do $\mathrm{VO}_{2}$ no LV $(43,6 \%)$ e $\mathrm{VO}_{2 \text { pico }}(15 \%)$. Com isso, concluiu-se que um regime de treinamento que melhore o desempenho no exercício não resulta necessariamente em mudanças na relação $\mathrm{VE}-\mathrm{VCO}_{2}$ de indivíduos saudáveis. Curiosamente, os sujeitos do trabalho de Clark et al. ${ }^{1}$ eram menos condicionados do que os indivíduos do presente estudo e o período de treinamento utilizado foi maior. Além disso, o protocolo do TCPE e a técnica de cálculo utilizada para determinação da EV no trabalho de Clark et al. ${ }^{1}$ e no presente estudo foram semelhantes. Desse modo, as diferenças entre os resultados de ambos os trabalhos não são claras.
Similarmente, Mourot et al. ${ }^{4}$ não encontraram alterações na EV de 15 mulheres saudáveis e ativas após seis semanas de treinamento intervalado composto por nove séries de quatro minutos na intensidade do LV, intercalados com um minuto na intensidade do $\mathrm{VO}_{2 \text { pico }}$ e realizado três vezes por semana. Apesar disso, houve um aumento de 6\% e 15\% no $\mathrm{VO}_{\text {2pico }}$ e $\mathrm{VO}_{2} \mathrm{LV}$, respectivamente. Em contraste com o presente estudo, Mourot et al. ${ }^{4}$ determinaram a EV por meio do slope VE-VCO ${ }_{2}$ calculado até o LV e razão VENCO ${ }_{2}$ no ponto do LV, o que limita comparações. Mourot et al. ${ }^{4}$ concluíram que diferentemente do $\mathrm{LV}_{\text {e }} \mathrm{VO}_{2 \text { pico, }}$ os índices de EV não são suficientemente sensíveis para destacar mudanças na capacidade cardiorrespiratória de indivíduos saudáveis. De fato, a magnitude da meIhoria da EV no presente estudo (i.e. redução de 4,1\% no slope VE-VCO $\mathrm{CO}_{2}$ ) foi pequena em relação ao aumento do $\mathrm{VO}_{2 \text { pico }}$ e $\mathrm{VO}_{2} \mathrm{LV}$. Isso evidencia que embora a EV seja uma medida reprodutível ${ }^{6,12}$, podendo ser utilizada de forma complementar na avaliação dos efeitos do treinamento, a sensibilidade é pequena quando se trata de indivíduos saudáveis.

Davies et al. ${ }^{18}$ demonstraram que existe um limiar crítico de capacidade física, abaixo do qual as anormalidades ventilatórias são evidentes. Eles verificaram que apenas quando o prejuízo da aptidão cardiorrespiratória excede um nível crítico de severidade, evidenciada por um $\mathrm{VO}_{2 \max }$ abaixo de $20 \mathrm{ml} . \mathrm{kg}^{-1}$. $\mathrm{min}^{-1}$ é que são encontrados prejuízos na EV. Essa situação é frequentemente observada em indivíduos com insuficiência cardíaca crônica severa, onde o débito cardíaco em resposta ao exercício é insuficiente para perfundir adequadamente todas as zonas pulmonares ventiladas ${ }^{18}$. $O$ achado interessante do presente estudo foi que mesmo em indivíduos saudáveis com boa tolerância ao exercício, o treinamento foi eficaz em provocar alterações na EV, uma medida que vem sendo mais utilizada na avaliação de sujeitos com algum prejuízo pulmonar ou cardíaco.

De modo geral, o slope VE-VCO 2 aumenta com a redução da $\mathrm{PaCO}_{2}$ e uma elevação da fração do volume do espaço morto (VD) ${ }^{17}$. Até a fase de tamponamento isocápnico a $\mathrm{PaCO}_{2}$ é regulada normalmente, permanecendo relativamente constante, portanto, quando um slope $\mathrm{VE}-\mathrm{VCO}_{2}$ aumentado é reportado até essa fase do exercício, isso ocorre provavelmente devido a um VD mais alto ${ }^{26}$. Após esse estágio, a acentuação do slope VE-VCO 2 é causada principalmente pela redução da $\mathrm{PaCO}_{2}$ devido a hiperventilação em resposta à acidose lática ${ }^{26}$. A utilização de uma fração anormalmente elevada do VD durante o TCPE (i.e. falha em reduzir a razão VDNT), vista na insuficiência cardíaca e em doenças pulmonares restritivas², contribui para a anormalidade do slope VE-VCO 2 desses sujeitos e ocorre como uma compensação para a baixa perfusão dos alvéolos ventilados ${ }^{3}$. Em contrapartida, em indivíduos saudáveis, a razão VDNT cai normalmente durante o TCPE, tendo em vista que o volume corrente (VT) aumenta substancialmente em relação ao VD, o que por sua vez, eleva a interação entre a ventilação alveolar e o fluxo sanguíneo capilar aumentando o potencial de troca gasosa ${ }^{17}$. Tem sido sugerido que um padrão respiratório mais profundo e mais lento, ocasionado por um aumento do volume corrente e/ou diminuição da frequência respiratória, reduz o componente resistivo da respiração, o VD e o custo de oxigênio da mesma contribuindo para a eficiência ventilatória ${ }^{27}$. Entretanto, tendo em vista que a alteração da razão VDNT em indivíduos saudáveis após o treinamento não é consistente ${ }^{4}$, parece que nesses sujeitos o principal mecanismo de aumento da EV seja um atraso no início da acidose lática, o que foi indiretamente confirmado no presente estudo pelo aumento do LV. É possível que esse atraso reduza a quantidade de lactato a ser tamponado diminuindo a resposta ventilatória. Além disso, tem sido sugerido que o aumento do LV com o treinamento retarda a queda da $\mathrm{PaCO}_{2}$ que ocorre em altas intensidades de exercício ${ }^{28}$, o que também pode atenuar a resposta ventilatória durante o TCPE, e contribuir para a redução do slope VE- $\mathrm{VCO}_{2}$.

Embora a melhoria da EV avaliada pelo slope VE- $\mathrm{VCO}_{2}$ em indivíduos saudáveis não seja um consenso ${ }^{1,4}$, a redução da ventilação durante o exercício submáximo de carga constante ${ }^{29}$ e da razão VE $\mathrm{NCO}_{2}$ em pontos 
do TCPE ${ }^{30}$, ambos em intensidades acima do LV, já foram demonstrados previamente nessa população. Casaburi et al. ${ }^{29}$ sugerem que essa redução da VE em cargas de trabalhos submáximas acima do LV é decorrente de alterações na capacidade aeróbia dos músculos locomotores, que levam a uma menor produção de lactato e a uma resposta hipercápnica atenuada, o que permite especular que o aumento do condicionamento cardiorrespiratório e metabólico leva a melhoria da EV.

Conforme esperado, os valores do slope VE- $\mathrm{VCO}_{2}$ dos indivíduos do presente estudo caracterizaram uma resposta ventilatória normal, ficando dentro dos valores encontrados na literatura para indivíduos saudáveis

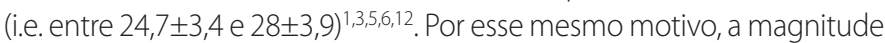
da redução no slope $\mathrm{VE}-\mathrm{VCO}_{2}$ foi pequena em relação ao que tem sido verificado em trabalhos utilizando indivíduos com IC crônica. Nessa população, a redução no slope $\mathrm{VE}-\mathrm{VCO}_{2}$ tem variado entre $6 \%$ a $23 \%$ após o treinamento aeróbio com duração de 3 a 24 semanas $8,10,13-16,19,20$. A implicação da melhoria da EV para esses sujeitos inclui principalmente a atenuação dos sintomas relacionados à intolerância ao exercício, o que tem sido atribuído à melhora da perfusão dos alvéolos ventilados em função de um aumento do débito cardíaco máximo, o que por sua vez reduz a fração do VD da ventilação ${ }^{16}$; atraso do início da acidose metabólica decorrente de um aumento na capacidade oxidativa dos músculos esqueléticos ${ }^{16,19}$ e a redução da quimiosensibilidade ${ }^{13,16}$

Para indivíduos saudáveis, é possível sugerir que um aumento da EV signifique um menor desconforto respiratório em altas intensidades de exercício, por meio da redução do impulso ventilatório mediado pela meIhora do equilíbrio ácido-base. No entanto, a relação direta da melhoria da EV com o aumento do desempenho de indivíduos saudáveis não é clara. A respeito disso, Brown et al.5,6 encontraram uma correlação fraca entre o slope $\mathrm{VE}-\mathrm{VCO}_{2}, \mathrm{VO}_{2 \max }{ }^{6}(\mathrm{r}=0,29)$ e $\mathrm{VO}_{2}$ no ponto de início de acúmulo de lactato em ciclistas $^{5}(r=-0,29)$, sugerindo que a EV parece ter uma importância limitada na avaliação do desempenho de atletas desta modalidade.

Por outro lado, em sujeitos que ainda não atingiram o limite superior do potencial de desempenho, como é o caso dos indivíduos do presente estudo, o aumento da EV em paralelo com o aumento do $\mathrm{VO}_{2 \text { pico }} \mathrm{eVO}_{2} \mathrm{LV}$, mostra que o slope VE- $\mathrm{VCO}_{2}$ é sensível à melhora do condicionamento, embora a magnitude seja pequena. Adicionalmente, apesar de a impor-

\section{REFERÊNCIAS}

1. Clark AL, Skypala I, Coats AJ. Ventilatory efficiency is unchanged after physical training in healthy persons despite an increase exercise tolerance. J Cardiovasc Risk. 1994;1(4):347-51.

2. Habedank D, Reindl I, Vietzke G, Bauer U, Sperfeld A, Gläser S, et al. Ventilatory efficiency and exercise tolerance in 101 healthy volunteers. Eur J Appl Physiol Occup Physiol. 1998;77(5):421-6.

3. Sun XG, Hansen JE, Garatachea N, Storer TW, Wasserman K. Ventilatory efficiency during exercise in healthy subjects. Am J Respir Crit Care Med. 2002;166(11):1443-8.

4. Mourot L, Perrey S, Tordi N, Rouillon JD. Evaluation of fitness level by the oxygen uptake efficiency slope after a short-term intermittent endurance training. Int J Sports Med. 2004;25(2):85-91.

5. Brown SJ. Cardio-respiratory system efficiency in trained endurance cyclists. Medicina Sportiva. 2010;14(4):176-81

6. Brown SJ, Raman A, Schlader Z, Stannard SR. Ventilatory efficiency in juvenile elite cyclists. J Sci Med Sport. 2012;16(3):266-70

7. Arena R, Myers J, Guazzi M. The clinical and research applications of aerobic capacity and ventilatory efficiency in heart failure: an evidence-based review. Heart Fail Rev. 2008;13(2):245-69.

8. Meyer K, Schwaibold M, Westbrook S, Beneke R, Hajric R, Görnandt L, et al. Effects of short-term exercise training and activity restriction on functional capacity in patients with severe chronic congestive heart failure. Am J Cardiol. 1996;78(9):1017-22.

9. Ingle L, Goode K, Carroll S, Sloan R, Boyes C, Cleland JG, et al. Prognostic value of the VENCO2 slope calculated from different time intervals in patients with suspected heart failure. Int J Cardiol. 2007;1 18(3):350-5.

10. Guazzi M, Reina G, Tumminello G, Guazzi MD. Improvement of alveolar-capillary membrane diffusing capacity with exercise training in chronic heart failure. J Appl Physiol. 2004;97(5):1866-73.

11. Buller NP, Poole-Wilson PA. Mechanism of the increased ventilatory response to exercise in patients with chronic heart failure. Br Heart J. 1990;63(5):281-3.

12. Chua TP, Clark AL, Amadi AA, Coats AJ. Relation between chemosensitivity and the ventilatory response to exercise in chronic heart failure. J Am Coll Cardiol. 1996; 27(3):650-7.

13. Van Laethem C, Van De Veire N, De Backer G, Bihija S, Seghers T, Cambier D, et al. Response of the oxygen uptake efficiency slope to exercise training in patients with chronic heart failure Eur J Heart Fail. 2007;9(6-7):625-9.

14. Coats AJ, Adamopoulos S, Radaelli A, McCance A, Meyer TE, Bernardi L, et al. Controlled trial of physical training in chronic heart failure. Exercise performance, hemodynamics, ventilation, and autonomic function. Circulation. 1992;85(6):2119-31.

15. Guazzi M, Vitelli A, Arena R. The effect of exercise training on plasma NT-pro-BNP levels and its correlation tância do aumento da EV em indivíduos saudáveis ser baixa em relação à relevância clínica que essa adaptação tem para pacientes com IC, O conhecimento das circunstâncias nas quais o treinamento pode reduzir o requerimento ventilatório durante o exercício pode ser útil na elaboração de estratégias de prescrição de exercícios em programas de reabilitação.

As possíveis limitações do presente estudo incluíram a ausência do grupo controle e de dados que permitissem uma comparação direta com os resultados encontrados considerando as características da amostra, o protocolo de treinamento e técnica utilizada para calcular EV. Além disso, apesar de um slope VE-VCO $\mathrm{CO}_{2}$ menor do que 30 ser amplamente aceito como uma resposta ventilatória normal $13,7,12$ e embora haja pontos de corte acima desse valor para definir o grau de prejuízo da EV7; não existem níveis que permitam classificar a EV dentro da faixa considerada normal (i.e. slope $\mathrm{VE}-\mathrm{VCO}_{2}<30$ ). Sugere-se que estudos futuros sejam conduzidos buscando avaliar a influência de diferentes categorias de aptidão aeróbia sobre os valores do slope VE-VCO 2 de indivíduos saudáveis, no intuito de verificar a possibilidade de desenvolver níveis de classificação para EV quando esta se encontra dentro do espectro considerado normal.

\section{CONCLUSÃO}

O presente estudo mostrou que um programa de treinamento de 13 semanas, delineado para melhorar a condição aeróbia, foi eficaz em aumentar a EV de indivíduos saudáveis. Essa melhoria da EV ocorreu em paralelo com o aumento do $\mathrm{VO}_{2 \text { pico }}$ e $\mathrm{VO}_{2} \mathrm{LV}$, embora em menor magnitude. Contudo, esses resultados sugerem que o slope da relação VE- $\mathrm{VCO}_{2}$ pode ser utilizado de forma adicional na monitoração dos efeitos do treinamento de indivíduos saudáveis, complementando a interpretação da integração cardiorrespiratória do TCPE.

\section{AGRADECIMENTOS}

Os autores agradecem à Coordenação de Aperfeiçoamento de Pessoal de Nível Superior - CAPES, pelo apoio financeiro (modalidade bolsa de mestrado).

Todos os autores declararam não haver qualquer potencial conflito de interesses referente a este artigo. with improved exercise ventilatory efficiency in patients with heart failure. Int J Cardiol. 2012:158(2):290-1 16. Davey P, Meyer T, Coats A, Adamopoulos S, Casadei B, Conway J, et al. Ventilation in chronic heart failure: effects of physical training. Br Heart J. 1992;68(5):473-7.

17. Wasserman, K, Hansen JE, Sue DY, Casaburi R, Whipp B. Prova de esforço: princípios e interpretação. 3aed. Rio de Janeiro, RJ: Revinter; 2005.

18. Davies, SW, Emery TM, Watling MI, Wannamethee G, Lipkin DP. A critical threshold of exercise capacity in the ventilatory response to exercise in heart failure. Br Heart J. 1991;65(4):179-83.

19. Kiilavuori K, Sovijärvi $A$, Näveri $H$, Ikonen $T$, Leinonen $H$. Effect of physical training on exercise capacity and gas exchange in patients with chronic heart failure. Chest. 1996;1 10(4):985-91.

20. Myers J, Dziekan G, Goebbels U, Dubach P. Influence of high-intensity exercise training on the ventilatory response to exercise in patients with reduced ventricular function. Med Sci Sports Exerc. 1999;31 (7):929-37.

21. Bruce RA, Kusumi F, Hosmer D. Maximal oxygen intake and nomographic assessment of functional aerobic impairment in cardiovascular disease. Am Heart J. 1973;85(4):546-62.

22. Howley ET, Bassett DR Jr, Welch HG. Criteria for maximal oxygen uptake: review and commentary. Med Sci Sports Exerc. 1995;27(9):1292-301

23. Novitsky S, Segal KR, Chatr-Aryamontri B, Guvakov D, Katch VL. Validity of a new portable indirect calorimeter: the AeroSport TEEM 100. Eur J Appl Physiol Occup Physiol. 1995;70(5):462-7.

24. Beaver WL, Wasserman K, Whipp BJ. A new method for detecting anaerobic threshold by gas exchange. J Appl Physiol. 1986;60(6):2020-7.

25. Caiozzo VJ, Davis JA, Ellis JF, Azus JL, Vandagriff R, Prietto CA, et al. A comparison of gas exchange indices used to detect the anaerobic threshold. J Appl Physiol. 1982;53(5):1184-9.

26. Sue DY. Excess Ventilation during Exercise and Prognosis in Chronic Heart Failure Am J Respir Crit Care Med. 2011;183(10):1302-10.

27. Neder JA, Dal Corso S, Malaguti C, Reis S, De Fuccio MB, Schmidt H, et al.The pattern and timing of breathing during incremental exercise: a normative study. Eur Respir J. 2003;21(3):530-8.

28. Lucía A, Carvajal A, Calderón FJ, Alfonso A, Chicharro JL. Breathing pattern in highly competitive cyclists during incremental exercise. Eur J Appl Physiol Occup Physiol. 1999;79(6):512-21.

29. Casaburi R, Storer TW, Wasserman K. Mediation of reduced ventilatory response to exercise afte endurance training. J Appl Physiol. 1987;63(4):1533-8.

30. Bhambhani $Y$, Singh $M$. The effects of three training intensities on $\mathrm{VO}_{\max }$ and $\mathrm{VE} / \mathrm{NO}_{2}$ ratio. Can J Appl Sport Sci. 1985;10(1):44-51. 